MS16-03

\section{Metal-organic frameworks with controllable arrangements of multiple metal cations}

Celia Castillo' ${ }^{1}$ Víctor Antonio de la Peña-O'Shea², Inés PuenteOrench $^{3}$, Enrique Gutierrez-Puebla ${ }^{1}$, Felipe Gándara ${ }^{1}$, M. Ángeles Monge ${ }^{1}$

1. Institut of Materials Science of Madrid (CSIC), Madrid, Spain

2. IMDEA Energy, Madrid, Spain

3. Institut Laue Langevin, Grenoble, France

email: castillo@icmm.csic.es

The combination of multiple metal elements within a same structure is highly desirable because the resulting materials might exhibit new or enhanced properties. Metal-organic frameworks (MOFs) offer a suitable platform to incorporate multiple metal elements; however multi-metal MOFs typically appear as solid solutions with lack of control over the distribution of the selected elements. We have developed a multi-metal MOF system based on a helical-shaped secondary building unit (SBU), which is able to incorporate zinc, manganese, cobalt, calcium and/or magnesium in a controllable manner. The combination of these elements at selected molar ratios results in the addressed realization of multi-metal arrangements with precise, atomic control over their location in the SBUs. Structural elucidation of the resulting elemental sequences is possible through the combination of different techniques including single crystal X-ray diffraction, neutron powder diffraction, electron microscopy and energy-dispersive X-ray spectroscopy.
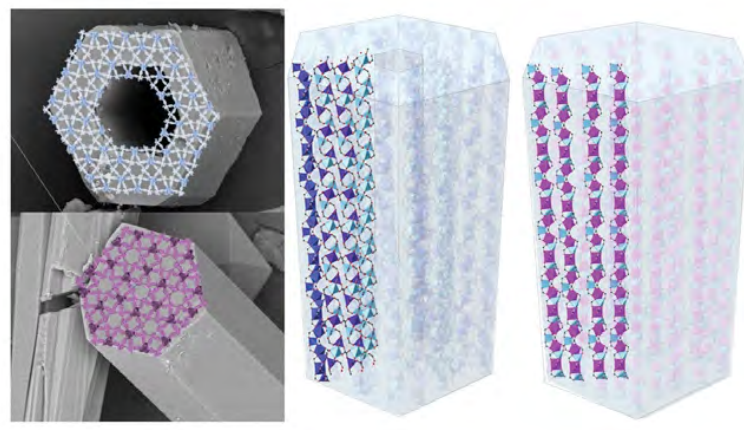

References:

Castillo-Blas, C., de la Peña-O'Shea, V. A, Puente-Orench, I., Romero de Paz, J., Sáez-Puche, R., Gutiérrez-Puebla, E., Gándara, F. \& Monge, M.A,. Science Advances. 2017, 3, 7, e1700773Keywords: MOFs, multication, characterization

Keywords: MOFs, multication, characterization

\section{VS16-04}

\section{Disorder-order transitions in the perovskite metal-organic frameworks $\left[\left(\mathrm{CH}_{3}\right)_{2} \mathrm{NH}_{2}\right]$ $\left[\mathrm{M}(\mathrm{HCOO})_{3}\right]$ at high pressure}

Ines Collings ${ }^{1}$, Maxim Bykov ${ }^{2}$, Elena Bykova ${ }^{3}$, Michael Hanfland ${ }^{1}$, Sander van Smaalen ${ }^{4}$, Leonid Dubrovinsky ${ }^{2}$, Natalia Dubrovinskaia ${ }^{4}$

1. European Synchrotron Radiation Facility, Grenoble, France

2. Bayerisches Geoinstitut, University of Bayreuth, Bayreuth, Germany

3. Deutsches Elektronen-Synchrotron, Hamburg, Germany

4. Laboratory of Crystallography, University of Bayreuth, Bayreuth, Germany

email: ines.collings@esrf.fr

The dimethylammonium metal formate compounds with the perovskite architecture have attracted interest over the years due to the coexistence of electric and magnetic orders [1], which in the case of the iron analogue, can be coupled [2]. The interesting physical properties are observed upon cooling, with a disorder-order transition at 160-270 K that originates from the loss of dynamic disorder of the dimethylammonium cation (DMA) $[1,3]$, and an antiferromagnetic ordering for the magnetically active $\mathrm{M}^{2+}$ cations below 35-8 $\mathrm{K}$ [1]. The hydrogen bonding interaction from the DMA cation to the formate can allow for magnetoelectric coupling within certain compositions [2]. However, the temperatures needed for the multiferroic behaviour in these materials are below $35 \mathrm{~K}$, and the coupling between the magnetic and electric orders are very weak. In an effort to explore different structural configurations with potentially improved physical properties, we study the dimethylammonium metal formates under pressure. In particular, we explore the possibility of inducing a disorder-order transition of DMA through reducing the volume using pressure. Similar structural configurations may then be replicated at ambient conditions by using "chemical pressure" through selection of different sized chemical components during synthesis.

High-pressure single-crystal X-ray diffraction is presented for the dimethylammonium metal formates (DMAMF), $\left[\left(\mathrm{CH}_{3}\right)_{2} \mathrm{NH}_{2}\right]\left[\mathrm{M}(\mathrm{HCOO})_{3}\right]$ where $\mathrm{M}=\mathrm{Mn}^{2+}, \mathrm{Fe}^{2+}$, and $\mathrm{Cu}^{2+}$, in order to compare the high-pressure phases with the known low-temperature ferroelectric (for DMAMnF) and multiferroic (for DMAFeF) phases. The ambient phases of dimethylammonium metal formates were stable up to 5.53(3), 5.7(3), and 7.3(2) GPa for DMAMnF, DMACuF, and DMAFeF, respectively. After these pressures, phase transitions occurred that were initiated by the structural distortion of the metal formate framework. The impact of the high-pressure distortions on the dynamic disorder of the dimethylammonium cation for DMAFeF and DMAMnF, and the Jahn-Teller distortion for DMACuF will be discussed. In the case of DMAFeF, the experiment was in addition conducted with a penetrating pressure-transmitting medium (PTM) that resulted in a different pressure-dependent behaviour due to the PTM inclusion.

References:

[1] Jain, P. et al. (2009), J. Am. Chem. Soc., 131, 13625-13627.

[2] Tian, Y. et al. (2014), Sci. Rep., 4, 6062-6066.

[3] Sánchez-Andújar, M. et al. (2010), Inorg. Chem., 49, $1510-1516$.

Keywords: perovskite metal-organic frameworks, high pressure, disorder-order transitions 